\title{
Predictive Projection in Color Fundus-Retail Images for Detection of Alzheimer's disease
}

\author{
P. S. Ramesh, S.Arivalagan, P.Sudhakar
}



\begin{abstract}
Alzheimer's disease is a prominent abnormality found in population above 50-60 years. This disease is prevalent in elderly resulting in steady decline of memories, functioning of social and motor abilities, cognition. This abnormality is identified by sedimentation of various types of proteins in brain of a human being. The reports are manually graded in conventional methods of treatments. It is also believed that manual analysis provides the exact measure of progress/regress of the disease. This research has been a primary case in medical history and it has been subjected to many investigations in recent decades. The prevalence of degradations has been identified in neuron networks of retina too. From the results investigated and modules derived by many researchers state, that there are distinct changes in parameters of human retina of Alzheimer's disease affected patients. Since these altered parameters could be utilized for detection of this disease, neuropathology advocated the usage of biomarkers to observe the diseases. Comparatively, these methods of monitoring retinal fundus images have proven to be non-invasive to the other methods, since retinal images provide a transplant medium for the studies. Managing this Alzheimer's disease has become easier with retinal in ageing technology and usual testing methods. All these methods in turn improved the lifestyle of $A D$ infected patients. They also do not have any invasive techniques, which added to the suffering of patients. Treatment should be planned according to have a better diagnosis. As the proverb says, 'Prevention is better than cure', early treatments reduce the prolonged effects of a disease. The treatment and diagnosis plan should be reliable and trustworthy when being cost efficient. When the disease is at its early phases, a systematic approach could be used to profile the parameters of humankind. Multiple image processing techniques are implemented in medical industry to simplify the practice. This paper proposes an innovative technique of modeling color fundus images into axis to determine the progress of neurological degradations. This approach segments the retinal images for grading monitoring and diagnosing Alzheimer's disease.

Index Terms: Alzheimer's Disease, Axis Method, Color Fundus, Retinal Imaging, Diagnosis
\end{abstract}

\section{INTRODUCTION}

In the elderly population, a serious complication of deterioration in functioning of motor, cognition and memory in human brain occurs. Due to the fact that neurons and its

Revised Manuscript Received on October 30, 2019

* Correspondence Author

Mr. Ramesh P S*, Research Scholar, Annamalai University, Chidambaram, India-608002.

Dr. S. Arivalagan, Assistant Professor, Department of Computer Science and Engineering, Annamalai University, Chidambaram, India-608002.

Dr. P. Sudhakar, Associate Professor, Department of Computer Science and Engineering, Annamalai University, Chidambaram, India-608002.

(C) The Authors. Published by Blue Eyes Intelligence Engineering and Sciences Publication (BEIESP). This is an open access article under the CC BY-NC-ND license (http://creativecommons.org/licenses/by-nc-nd/4.0/) network are affected inside the brain, long term and life's events are erased which also affects the concentration and ability of doing a series of activities [1, 2]. Alzheimer's disease is paced as top 6th fatal diseases among other diseases and almost accounts for $34^{\text {th }}$ of dementia affected cases [3]. Another alarming warning to the humankind is that a person is getting affected by Alzheimer's disease every 70 seconds and this rate is increasing by its double every decade. This is to be affecting the age group above 65 years. The affected count throughout the world is almost about 38 million people by Alzheimer's disease or other variants of dementia. At this rate of growth, the affected group of people will be doubled in next ten years and might reach 120 million affected people by 2050 [4]. The development phases are categorized into four stages of Alzheimer's. Very first change of Alzheimer's is called as Mild Cognitive Impairment (MCI), which does not have a huge impact by altering just a minimal set of everyday actions. These next stages are classified into mild and moderate variants of Alzheimer's disease. These phases will display a notable change in cognitive shortfalls and would demand attention and proper care. The last stage is the server $\mathrm{AD}$ where the patients cannot be independent and have to rely on caretakers. They tend to lose their original Sanity and self [5]. In medical industries, dementia is the term used to mention the decline of thinking ability and functioning abilities making live difficult without proper medical attention. Alzheimer's disease has been listed as the primary type of dementia. The symptoms and results of this disease are inability to function normally, loss of memory, loss of judgmental ability, confusions, lost routine functions and weakening of brain. These symptoms are found to be common in Parkinson's disease, Frontotemporal dementia, vascular, lewy bodies' dementia. This will be processing a method to narrow down to Alzheimer's disease with the discussion of following symptoms.

1. Disorientation

2. Difficulty in speaking and identifying the meaning of common words even in a native language.

3. Cortical dysfunction

4. Agnosia- which is the difficulties in recognizing common objects, friends and relatives.

5. Disabled and difficult motor functions and most importantly losing memory.

After the fourth phase of $\mathrm{AD}$, the patients and bedridden due to disability of limbs completely. The brain structure and anatomy is completely affected and many reports have shown distorted cortical atrophy and enlargement of veins and vessels. The distinguishing factor of $\mathrm{AD}$ is the presence of abnormities inside and outside the neuron. Neurons are filled with senile plaques and outside the neurons, neurofibrillary tangled structures are formed.

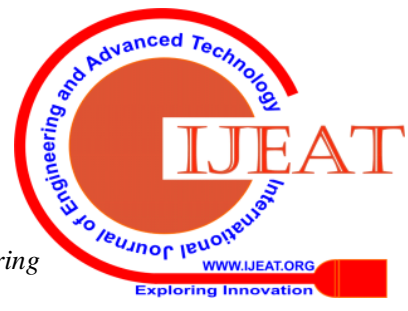


These tangles are formation of cluster or bundled cytoplasm when neurons displace within the brain or nuclease becomes encompassed. On the other hand, senile plaques are represented as circular formations of amyloid beta cores. This is formed due to the improper processing of Amyloid Precursor protein (APP) [6, 7]. Imaging techniques are computerized for retrieving the reports in Computed Tomography Scans (CT), Magnetic Resonance Imaging (RETINAL FUNDUS), further to identify the exact abnormalities using tests like Mini Mental State Examination, Trail making tests A\&B, Digit and Symbol Matching Tests, Spanning forward and background of digits, Audio based verbal learning Test Fluency of every category and drawing a clock test [8]. All of these investigations are used to categorize the phase and analyze the level damage to brain. The patients are grouped based on their ability to recall realize and perceive the knowledge after narrowing down to impact, in search for a less invasive method for detecting $A D$, many proposal were put in and some identified the relationship of retinal damages to the disease. This was a breakthrough to analyze the transparent medium through minimal invasive medical devices [9]. The abnormal features of human eyes have been helpful to relate important of brain and dementia. The relationship between eye defects motor cognition and verbal deficiencies led to the adverse effects of AD or dementia.

Fundus cameras have been used to capture the retinal images and classify the grade of disease with captured features. These cameras are capable of analyzing the eyes based on sensitivity, specificity to quality vascular damage, retinopathy, maculopathy, changes in geometry, symmetrical properties and formation of eye vessels $[10,11]$. The pictures are delivered in digitized format, which easily implements techniques of automated image processing. The reliability, accuracy and speed of identification significantly improve when compared to that of traditional methods. Retina and vascular structure of the eyes are preserved in digital format for reuse, sharing and minimal storage efficiencies. This proposal intends to find a unique solution for analysis of AD using colour fundus imaging. For detection and prognosis, fundus images of $\mathrm{AD}$ patients are utilized for investigations and demonstration.

\section{BACKGROUND STUDY}

Processing of color fundus images include image acquisition, textures and features extraction, classification based on present features and segmentation. The knowledge of various domains in computer science is also applied with image processing techniques to process the obtained fundus images. Segmentation is the primary process for knowledge extraction. In one approach, techniques used in artificial intelligence (AI) is applied on image for achieving segmentation and partition of images. The preferred techniques in AI ia applied on images for achieving segmentation and partition of images. The preferred techniques in AI are fuzzy logic and neural networks when medical images have been processed [12]. Wavelet network is another commonly utilized method for this process of segmentation. The tasks of a wavelet network are listed as denoising, background removal and subtraction, highlighting features, characteristic and neural network capacity for generation and conversion into numerical description [13].this becomes the easier method of conversation and in terms of operations. The limitations of many previous techniques are addressed in wavelet networks.

Techniques and devices in Image Processing, especially for retina capturing has assisted the picturisation of human retina with a structural and functional presentation. With this advent, practitioners have been provided a chance of early detection, monitoring, progress, clinical trials in Alzheimer's disease. Ocular fundus otherwise called as retinal photography is the conventional method of retinal imaging which also facilitates periodical checkups. This technique categories the image into three types based on retinal vascular signs. They are

1. Qualitative retinopathy

2. Changes marked in caliber

3. Global geometry and symmetry of regular eye.

The qualitative and quantitative analysis of retinal vascular structures and grades of vascular reduction, enhanced vessel width, branching, reduction in optimality of geometrical details map to poor and affected cognitive performance. In other study by Rotterdam. The association of retinopathy and maculopathy with $\mathrm{AD}$ showed that they are more chances due to diabetes. Using CRAE index for vascular calibers, have demonstrated the reduction in width of arteries in $\mathrm{AD}$ affected patients. Micro vascular width and attenuation were derivations a subjected in standard deviation derivations and indicated that arterior and venular calibers have reduced. The fractal dimensions of geometrical patterns and fortuity found in $\mathrm{AD}$ affected patients are found to be common formats to confirm the link between AD and retinal photography [14].

The fundus images of AD patients displayed different patterns of RNFC with respect to diffusion and wedge shaped dropouts. Available software's for measuring retinal structure are not completely automated. Calibers, torusity, network complexity and other ocular fundus features are yet to be found accurately in computerized methodologies. In mydriatic ultra-wide field retinal imaging techniques, human eye is captured in a single picture that explains the vascular structure a retina for analyzing lesions much better than previous techniques [15]. In pattern electro-retinogram imaging techniques, estimator the ganglion and axons electrical response with pattern based inputs. ERG responses in $\mathrm{AD}$ patients have been reduced in amplitude and attenuation. The ganglion all malfunction also, maps to structural change in RNFC of AD patients. EKG results were not constant and returned normal results even in mild AD patients. PERG are preferred during the starting phases of AD. This technique is time consuming and preferred with more availability. Retinal oximetry is a technique of measuring saturated levels of oxygen in blood vessels by a non-invasive, fast and safer methodology. This follows a spectrophotometric fundus imaging. The metabolic and functional abilities of retina will be reflected by repeated analysis of oxygen saturation in retinal blood vessels. This technique has facilitated detection of numerous diseases due to retinal malfunctions, diabetics, maculopathy and even brain diseases [16].

Published By: 
This enabled the usage of RO technique for detecting AD. Doppler OCT was proposed to represent the flow of blood and measures to qualify them in vivo. Patients with AD are found to have congested retinal blood vessels that limit the normal flow of blood to eyes and brains. This reduction in retina and brach affects the APP synthesis resulting in death of neurons because of less than expected blood flow. Through the measure of these changes in ocular vascular columns, monitoring AD was simplified $[17,18]$.

Yet another non-invasive technique in fundus photography is retina micro-perimetry. This is a simple and fast technique which measures and highlights the light sensitivity of macular regions. Irrespective of eyeball movements, this is most preferred technique for monitoring AD. The brain's neurodegeneration and retinal sensitivity to light can be related with each other and gives the prediction of how diabetes patients can be prove to $\mathrm{AD}$. This technique has also outperformed ERG in identifying functional changes. This method is also cost affective application for monitoring the progress of AD.

\section{ANATOMY OF HUMAN EYE}

Human eye is a sensory organ receiving light and act in response to provide vision. Dynamic objects in daylight are projected as a three dimensional perception to humans after processed by the human eyes. The perception is defined by an entire segment of the brain for deriving information about this world. Working as a camera, the eyes process light reflected from objects into meaningful information leading to further actions directed from brain. Motivated from operations of human eye, the light rays reflected from objects enter into a lens and falls onto a magnetic film. When the magnetic film is processed chemically, the pictures will retain the information permanently. Likewise, light rays enter the pupil and falls onto a special layer in the back of eye called retina. The retina sends the image to the brain for processing the picture. Science says that the human eye is capable of differentiating millions of colors and can even detect a single photon [15]. Yet, the eyes are more advanced in focusing on a single object of diverse sizes, luminosity and contrast, which is not possible even in high tech cameras nowadays. The components of a normal human eye are portrayed in Figure 1. The very first layer of human eye is the cornea, acting as the gateway of light to pass into. The cornea filters the rays of light entering into eye after focusing on the particular object. The eye seems rigid and slightly arched from the front, and this is due to a glutinous and thick substance named aqueous humour in the anterior chamber. The light with varying brightness may lead to unwanted effects and this is balanced by contracting and relaxing the pupils. When we tend to enter from a bright room into darkness, the eye's muscles called iris, expands to facilitate more light to provide some vision. And on the other way around, the expanded iris will produce a glaring effect when we see a bright light exiting from a dark room. This balancing will be limiting and permitting the amount of light entering into the eyes. This action is to enable the rays of light to fall onto the retina, for analysis by the brain. This research work will be concentrating on retinal structures and associated anatomical features for this automated system.

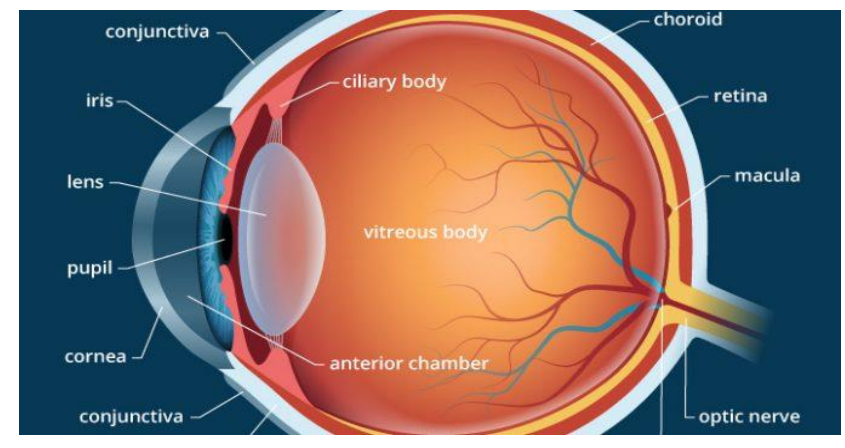

Fig.1 Human Eye Anatomy

\section{PROPOSED SYSTEM}

The proposed methodology involves a series of preproduction processing which assist in reducing the area of analysis. This method is different from other proposed methods as techniques like Image processing and Background separation or subtractions have been used. Background separation is used to remove the unwanted factors surrounding the area to be analyzed, which enables easy detection of the abnormalities. Similarly, the image of the eyes beside the affected area need not be analyzed. When the region of abnormalities are detected and cured, eventually the surrounding parts are alleviated. The infected area alone can be differentiated by means of perimeter and area constraints of image processing techniques. Image processing techniques are the most powerful and sophisticated method which easily renders information from the image. As position, texture and intensity is considered to be as the major factors, Image processing techniques yields the exact information about these attributes. The resultant parameters render the characteristics of the abnormalities and helps in identifying the definite position of the abnormalities. This method is highly discriminating from other abnormalities detection methods.

\section{Background Separation}

Background separation is method of separating the image and background. The images are obtained as a square form, with meaningless black matter surrounding the gray matter in the middle. The gray matter represents the structure of the brain, along with the abnormalities in specified regions in different shapes and sizes. Recorded results show a affected region as a deformed circle or abnormal circle like shapes. The first step is to eliminate the black matter and unaffected space of the brain from the abnormalities regions.

By this, the resultant set of images to be subjected for analysis into the automated system would be regions of abnormalities. Separation of such images would render to save execution time to finalize the decision. Thresholding is the simplest method of image segmentation. Using a parameter to distinguish the variance between different pixels, thresholding attempts to divide the images into smaller portions after marking a boundary. Such a parameter may be considered to be $40 \%$ portion of gray in a grayscale image: pixels found to exceed this $40 \%$ gray parameter may be classified into a segment, and the rest are accommodated to the next segment. 
This is the primary process of image processing. When there are considerable and distinct features in both foreground and background, thresholding is applied to overcome the visibility issues. From a gray scale image, binary images are derived from thresholding processes. Thresholding is one of a notable process in the series of background separation. The retinal image comprises of two colors- red, orange, black and gray (nearly white) colors for differentiation of healthy and affected regions. Thresholding algorithm tends to eliminate the background from the foreground of specified intensity or color information. Thresholding method is assumed that the intensity values are different in different regions. A threshold is said to be globally optimal if the number of misclassified pixels is minimum. The resultant output can be of two forms. The output image could be either obtained as the foreground or background alone. A parameter is the comparative element to be altered with the pixel of input images and altered with the intensity parameter on proving the condition true or false. The diversity of factors affect the images like light effects, changes due to motions and background changes which are addressed by a subtraction algorithm. A function of $\mathrm{V}(\mathrm{a}, \mathrm{b}$, and $c$ ) is used for analyzing the video sequence where $c$ denotes time dimension, $\mathrm{a}$ and $\mathrm{b}$ denote pixel location variables.

\section{Analyzing the Difference between Consecutive Frames}

Exact difference between two frames of a particular region at any given time $t+1$ is obtained with the following equation.

$$
\mathrm{D}(\mathrm{c}+1)=|\mathrm{V}(\mathrm{a}, \mathrm{b}, \mathrm{c}+1)-\mathrm{V}(\mathrm{a}, \mathrm{b}, \mathrm{c})|
$$

Frame represents both foreground and background features and the background is identified as the frame at time t. Subtraction from two frames will represent the changes in intensity of the pixel locations that is subtracted from two frames. This technique is found to work successfully for images that have features that are in motion and a stable background images. When subtraction is applied to already existing processed images, the quality improves with the following equation.

$$
|\mathrm{V}(\mathrm{A}, \mathrm{B}, \mathrm{C})-\mathrm{V}(\mathrm{A}, \mathrm{B}, \mathrm{C}+1)|>\mid \text { MATHRM }\{\mathrm{TH}\} \backslash
$$

\section{Mean filter}

When images are identified for possess only background features, previous images captured from similar conditions are approximated for a referential objective. The background image is implied with the following equation with instant value of $c$,

$$
B(a, b)=\{1 \backslash \text { over } N\} \backslash \text { sum_ }\{i=1\} \wedge N \text { V }(a, b, c-i)
$$

$\mathrm{N}$ denotes the number of images from previously matching conditions. $\mathrm{N}$ will also impose the speed of video and frames played in given time. Computing the background $\mathrm{B}(\mathrm{a}, \mathrm{b})$ is followed by subtraction of one from the image $\mathrm{V}(\mathrm{a}$, $\mathrm{b}, \mathrm{c})$ at time $\mathrm{c}=\mathrm{t}$. Thresholding is applied to those images and derives the foreground.

$$
\mid \mathbf{V}(\mathbf{a}, \mathbf{b}, \mathbf{t}) \text { - B(a, b) }|>| \text { mathrm }\{\mathrm{Th}\} \backslash,
$$

Th denotes the threshold. Mean is observed from the previous equation and computed with $\mathrm{B}(\mathrm{a}, \mathrm{b})$.

\section{Processing Gaussian Averages}

Wren et al. invented the utilization of a Gaussian probabilistic density function (pdf) on the latest $\mathrm{n}$ frames. Pdf form of an image is computed with a new time $t$, this process is supported by online cumulative average. The pdf of every pixel is characterized by mean \mu_t and variance \sigma $\wedge$

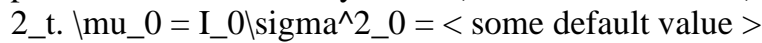

In which I_t denotes the value of the pixel's intensity at any given time $t$. Initializing the variance use the $\mathrm{x}$ and $\mathrm{y}$ from a small window around each pixel. Many factors influence the changes in images like illumination changes or non-static background objects. This difference in every frame $t$ the pixel's mean and variance must be updated with the following equation.

$\backslash \mathrm{mu}$ _t $=\backslash$ rho I_t $+(1-\backslash$ rho $) \backslash \mathrm{mu} \_\{\mathrm{t}-1\}$

$\backslash$ sigma $\wedge 2 \_t=d \wedge 2 \backslash$ rho $+(1-\backslash$ rho $) \backslash \operatorname{sigma} \wedge 2_{-}\{\mathrm{t}-1\}$

$$
\mathrm{d}=\mid\left(\mathrm{I} \_\mathrm{t}-\left\langle\mathrm{mu} \_\mathrm{t}\right) \mid\right.
$$

\rho is used to determine the size of temporal window that is used to fit the pdf and d is the Euclidean distance between the mean and the value of the pixel.

\section{Pixel wise Gaussian distribution}

The pixel levels of in image's background are computed when its current intensity falls within some confidence domains using the equation.

$\backslash$ frac $\{\mid($ I_t $-\backslash$ mu_t $) \mid\}\{\mid$ sigma_t $\}>k \backslash$ longrightarrow $\backslash$ mathit $\{$ Foreground $\}$

frac $\left\{\mid\left(\mathrm{I} \_\mathrm{t}-\backslash\right.\right.$ mu_t $\left.) \mid\right\}\{\backslash$ sigma_t $\} \backslash$ le $\mathrm{k} \backslash$ longrightarrow Imathit $\{$ Background \}

$\mathrm{k}$ is a free threshold parameter assumed to be 2.5. For a greater dimensionality of background images produces a bigger $\mathrm{k}$, and it increases the probability of a conversion from background to foreground. The method varies with pixels distribution. Then they are updated and finally segmented as background. This eliminates newly captured foreground and are not affected by processes. The updated formula to determine the mean is as follows.

$\backslash \mathrm{mu} \_\mathrm{t}=\mathrm{M} \backslash \mathrm{mu} \_\{\mathrm{t}-1\}+(1-\mathrm{M})\left(\mathrm{I} \_\mathrm{t} \backslash \mathrm{rho}+(1-\backslash \mathrm{rho}) \backslash \mathrm{mu} \_\{\mathrm{t}\right.$ - 1\})

Here the value of $M=1$ when $I \_t$ is termed to be the foreground which is found to be constant and $M$ is 0 otherwise. The determination of background and foreground is decided by the value of intensity. The boundary levels are differentiated with varying intensity values.

\section{Models with Background Mixtures}

This technique is applied for every pixel in images and frames of the videos using Gaussian filters. The frequency of different intensities is obtained by a simple heuristic methodology. Pixels found to be unique are considered to be foreground pixels and the rest are considered to be background pixels.

Foreground pixels can be collectively formed using 2D connected component analysis. At any time t, a particular pixel (a_0, b_0)'s history is

$\mathrm{X} \_$1, \ldots, $\mathrm{x} \_\mathrm{t}=\backslash\left\{\mathrm{V}\left(\mathrm{a} \_0, \mathrm{~b} \_0, \mathrm{i}\right): 1 \backslash\right.$ leqslant $\mathrm{i} \backslash$ leqslant $\left.\mathrm{t} \backslash\right\}$

This history is modeled by a mixture of K Gaussian distributions:

$P\left(X \_t\right)=\backslash$ sum_ $\{i=1\} \wedge K \backslash$ omega_ $\{i, t\} N \backslash$ left $\left(X \_t \backslash\right.$ mid $\left.\backslash \mathrm{mu} \_\mathrm{i}, \mathrm{t}\right\}, \backslash$ Sigma_$\{\mathrm{i}, \mathrm{t}\} \backslash$ right $)$

Where,

$\mathrm{N} \backslash \operatorname{left}\left(\mathrm{X} \_\mathrm{t} \backslash \mathrm{mid} \backslash \mathrm{mu}\{\right.$ it $\}, \backslash$ Sigma_\{i, t $\} \backslash$ right $)=$ $\backslash \operatorname{dfrac}\{1\}\{(2 \backslash \mathrm{pi}) \wedge\{\mathrm{D} / 2\}\}\{1$ lover $\mid \wedge$ Sigma_ $\{\mathrm{i}, \mathrm{t}\} \mid \wedge\{1 / 2\}\}$ lexp\left(-\{1 lover 2$\}\left(\mathrm{X} \_t-\backslash m u \_\{\mathrm{i}, \mathrm{t}\}\right)^{\wedge} \mathrm{T} \backslash$ Sigma_\{i, $\mathrm{t}\} \wedge\{-1\} \backslash$ left (X_t $-\backslash \mathrm{mu} \_\{\mathrm{i}, \mathrm{t}\} \backslash$ right $) \backslash$ right $)$

An on K-means approximation is found to improve the Gaussians. 
The numerous developments of this actual method developed by Stauffer and Grimson have been proposed and a complete survey can be found in Bouwmans et al. Background subtraction is a computational vision process of extracting foreground objects in a particular image. A foreground image can be defined as an object of attention that helps in reducing the amount of data to be processed as well as providing the information to the task under consideration. Often, the foreground object can be thought of as a coherently moving object in a scene. It has to be emphasized the word coherent here because if a person is walking in front of moving leaves, the person forms the foreground object while leaves though having motion associated with them are considered background due to its repetitive behavior. In some other cases, the distance of the moving object also forms a basis for it to be considered a background, e.g in an image, one person is close to the camera while there is a person far away in background. In this, the nearby person is considered as foreground while the person far away is ignored due to its minimum size and the lack of information that it provides. Identifying moving objects from a video sequence is a fundamental and critical task in many computer-vision applications. A common approach is to perform background subtraction, which identifies moving objects from the portion of video frame that differs from the background model. Background subtraction is a technique for segmenting out objects of interest in a scene for applications such as surveillance and etc. There are lot of challenges in developing a fine background subtraction algorithm. First, it must be robust against changes in illumination of the image. Second, it should avoid detecting non-stationary background images and shadows cast by moving images. A good background model should also react quickly to changes in background and adapt itself to accommodate changes occurring in the background such as moving of a stationary chair from one place to another. It should also have a good foreground detection rate and the processing time for background subtraction should be real-time.

\section{Image Subtraction}

Image subtraction is a process whereby the digital numeric value of one pixel or whole image is subtracted from another image. This can be done for one of two reasons a) Leveling uneven sections of an image such as half an image having a shadow on it and b) Detecting changes between two images. This identification of changes can be used to inform if something in the image is moved. It is now possible to immediately subtract an image from the desired image in image processing techniques. An image subtraction algorithm is used in this project to recognize a moving object. The major reason for using this algorithm is that it is simple and can be implemented by the limited real-time processing capabilities of the image processing board. It turned out to work acceptably well. A single pass command have been devised to subtract the unwanted for or background regions in an image. Supplied with two images and pixel information of scalar (black and white) images, over the single pass, resultant image would readily display the regions useful for an analysis by a pathologist.
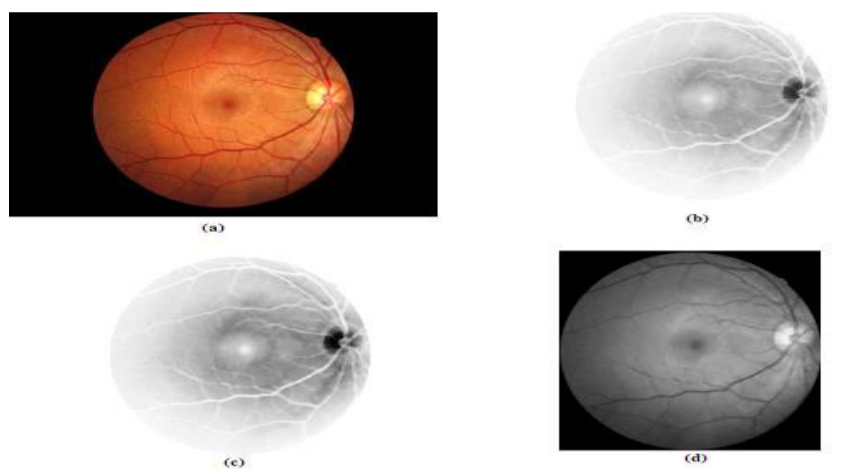

Fig. 2: (a) Color Fundus Image of affected eyes, (b) Conversion into grayscale image for easier processing, (c) Identifying the affected region, (d) Measuring by Referential Axis

The subtraction is explained as follows.

If the results are desired in pixel information,

$$
\mathrm{Q}(\mathrm{i}, \mathrm{j})=\mathrm{P}(\mathrm{i} 1, \mathrm{j} 2)-\mathrm{P}(\mathrm{i}, \mathrm{j})
$$

Rather, the images could subtracted to display the absolute differences,

$$
\mathrm{Q}=|\mathrm{P}(\mathrm{i} 1, \mathrm{j})-\mathrm{P}(\mathrm{i} 2, \mathrm{j})|
$$

Similarly, a constant value could be subtracted from the input image.

$$
\mathrm{Q}=\mathrm{P}(\mathrm{i} 1, \mathrm{j})-\mathrm{C}
$$

A retinal fundus image composed of a darker background and brighter foreground, after processing in the thresholding algorithm produces an intermediate result of inverted colored image. The example of thresholding clearly differentiates the area of the human brain or merely the color schemes. The same result can be subtracted by using any of the above mentioned schemes to eliminate the unwanted areas before inserted into the Referential Growth Points algorithm. Optimal method to subtract an area is to eliminate the boundary values of pixels into a smaller area of affected regional space. Removing the constant of black colored pixels may be removing the abnormalities region, which is inversed into black color itself (After Thresholding algorithm). A sample coding offers a better understanding.

$$
\begin{aligned}
& \mathrm{P} 1=\text { uint8([ } 2551075 ; 44225 \text { 100]); } \\
& \mathrm{P} 2=\text { uint8([ } 5050 \text { 50; } 505050 \text { ]); } \\
& \mathrm{Q}=\operatorname{imsubtract}(\mathrm{X}, \mathrm{Y}) \\
& \mathrm{Q}=2050 \quad 25 \\
& \quad 0175 \quad 50
\end{aligned}
$$

The boundary metrics can be represented in arrays, constituting an image, and could be subtracted from the intermediate output image of thresholding algorithm. After the two consecutive steps of preprocessing the input retinal fundus image of an affected patient would resemble the following image. The abnormalities have been clearly visible after the processing proposed in this research work. The border line would be space which has been left out after assigning the pixel values into the algorithm.

\section{RESULTS AND DISCUSSION}

The experimental analysis is the comparison of two images i.e., the healthy level and the affected level. 
This differentiation shows how far the abnormalities have increased in size than the healthy eye that is taken as the reference model. It is clearly seen that the patient reports has increased in size, eventually leads to increase in the intensity, the size and the shape. Both the abnormalities are segmented into divisions and analysis is further carried out. This segmentation leads easy retrieval of information in the identification of abnormal retinal images. Graphs are plotted for the respective tumor levels in accordance with the growth of the tumor. The growths of abnormal circles in horizontal and vertical coordinates are shown in Figure 3.
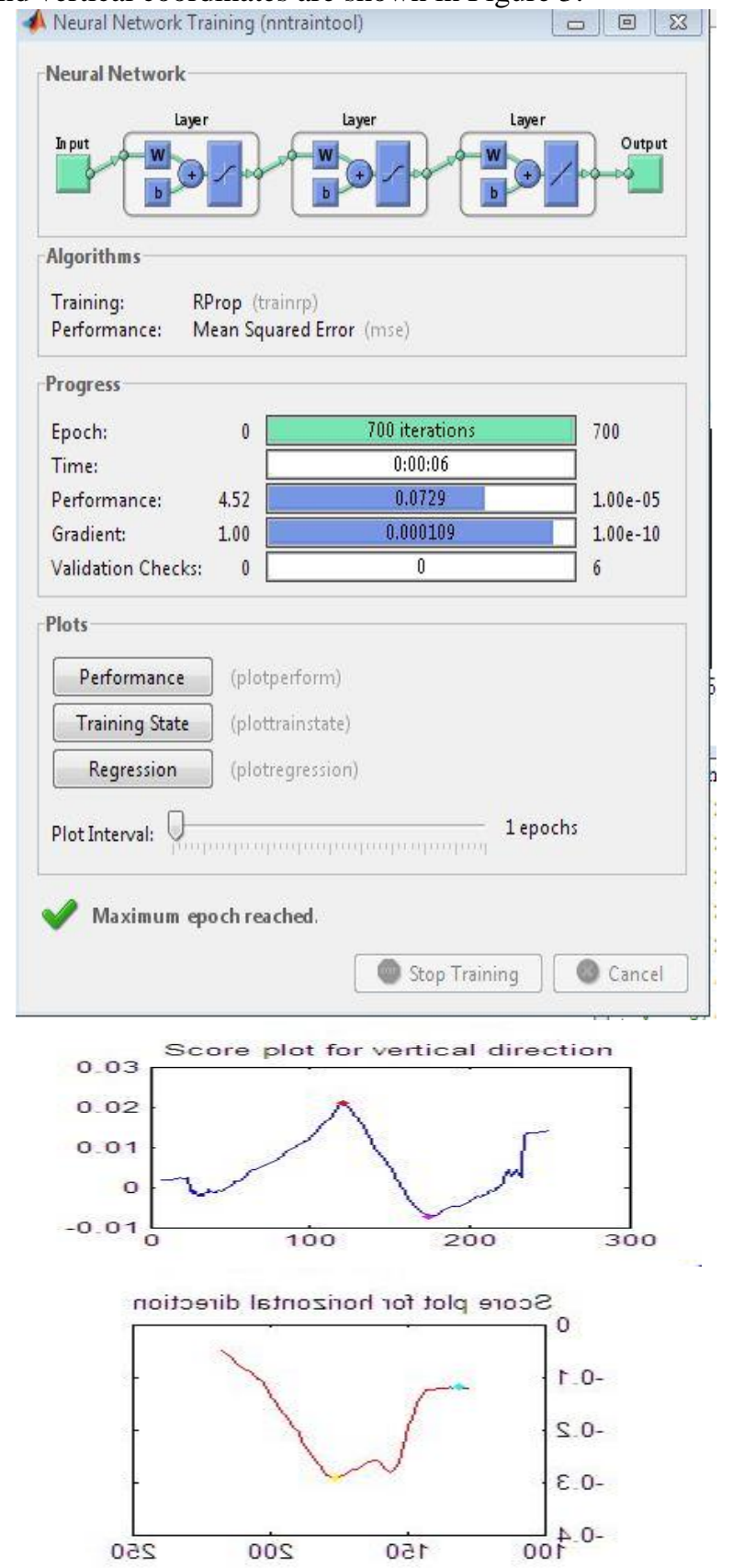

Fig. 3: Horizontal And Vertical Scale Plot For Second Tumor Image

The given graphs show a display of how the healthy eye and affected eye characteristics differ with respect to each other. These graphs give the comparison of the various levels of the affected eyes. These graphs help in identifying the increase and decrease of the affected regions with respect to progress of Alzheimer Disease level. The graphs are plotted in terms of bar chart, line chart and pie chart so that the progress ratio can be easily determined. The referential matrix proposed in this study is tabulated in Table 1 and Figure 4.

Table 1: Reference Table For Marking The Affected Regions

\begin{tabular}{|c|c|c|}
\hline $\begin{array}{c}\text { Referential } \\
\text { Point }(0-\mathrm{x}, 0+\mathrm{y})\end{array}$ & $\begin{array}{r}\text { Referential } \\
\text { Point }(0-\mathrm{x}, 0)\end{array}$ & $\begin{array}{r}\text { Referential } \\
\text { Point }(0-\mathrm{x}, 0-\mathrm{y})\end{array}$ \\
\hline $\begin{array}{c}\text { Referential } \\
\text { Point }(0+\mathrm{x}, 0+\mathrm{y})\end{array}$ & $\begin{array}{c}\text { Standard } \\
\text { Point }(0,0)\end{array}$ & $\begin{array}{r}\text { Referential } \\
\text { Point }(0-\mathrm{x}, 0-\mathrm{y})\end{array}$ \\
\hline $\begin{array}{c}\text { Referential } \\
\text { Point }(0+\mathrm{x}, 0+\mathrm{y})\end{array}$ & $\begin{array}{c}\text { Referential } \\
\text { Point }(0+\mathrm{x}, 0)\end{array}$ & $\begin{array}{c}\text { Referential } \\
\text { Point }(0+\mathrm{x}, 0-\mathrm{y})\end{array}$ \\
\hline
\end{tabular}

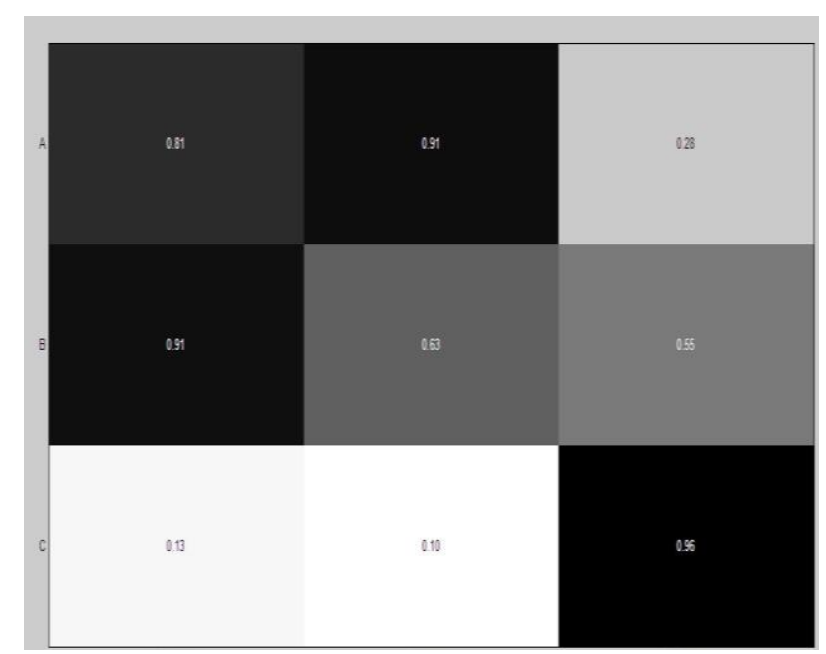

Fig. 4: Referential Matrix for affected regions

\section{CONCLUSION}

Color Fundus images of human retinal regions have given a simple yet efficient medium for identifying the progress of Alzheimer's disease. This method is invasive and requires minimal time for computing the rate of growth of an abnormal spill or sedimentation of proteins. The results and discussions have demonstrated the simplified process associated with grading the Alzheimer's disease. Time taken for computation is reduced when processing retinal images in its grayscale conversion. The consideration of RGB channels is considered as the directive measure. The reference matrix is equipped with abundant training set information for efficient comparisons. The matrix model will indicate the growth of affected regions from the axis. The methods used for differentiating the retina and affected regions are considered from the surveyed methods. Background separation and subtraction techniques define the exact regions to be concentrated. This proposed method acts as a useful tool with an optimized algorithm. Texture, color based analysis were implemented to differentiate the regions marking the affected circles into a matrix representation have been used to transform digital information into numerical information.

Published By:

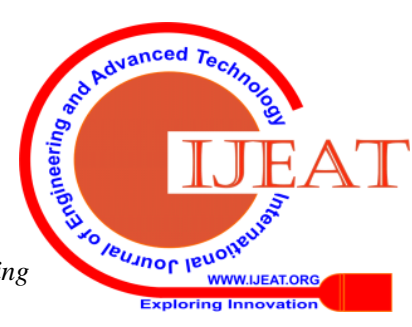


The features which satisfies the conditions defined by thresholds will be used for classifications and detection of Alzheimer's diseases.

\section{REFERENCES}

1. Sandeep, C. S., \& iSukesh Kumar, A. (2013). A Review Paper on the Early Diagnosis of Alzheimer's Disease (AD) through Profiling of Human Body Parameters. Scientistlink, Coimbatore, India, 2013, International Journal of Computer Science and Engineering Communications (IJCSEC), 1(1), 21-29.

2. Abràmoff, M. D., Lou, Y., Erginay, A., Clarida, W., Amelon, R., Folk, J. C., \& Niemeijer, M. (2016). Improved automated detection of diabetic retinopathy on a publicly available dataset through integration of deep learning. Investigative ophthalmology \& visual science, 57(13), 5200-5206.

3. Ascaso, F. J., Cruz, N., Modrego, P. J., Lopez-Anton, R., Santabárbara, J., Pascual, L. F., ... \& Cristóbal, J. A. (2014). Retinal alterations in mild cognitive impairment and Alzheimer's disease: an optical coherence tomography study. Journal of neurology, 261(8), 1522-1530.

4. Bayhan, H. A., Aslan Bayhan, S., Celikbilek, A., Tanık, N., \& Gürdal, C. (2015). Evaluation of the chorioretinal thickness changes in A lzheimer's disease using spectral-domain optical coherence tomography. Clinical \& experimental ophthalmology, 43(2), 145-151.

5. Cordeiro, M. F., Guo, L., Coxon, K. M., Duggan, J., Nizari, S., Normando, E. M., ... \& Moss, S. E. (2010). Imaging multiple phases of neurodegeneration: a novel approach to assessing cell death in vivo. Cell death \& disease, 1(1), e3.

6. Cheung, N., Mosley, T., Islam, A., Kawasaki, R., Sharrett, A. R., Klein, R., ... \& Wong, T. Y. (2010). Retinal microvascular abnormalities and subclinical magnetic resonance imaging brain infarct: a prospective study. Brain, 133(7), 1987-1993.

7. Csincsik, L., MacGillivray, T. J., Flynn, E., Pellegrini, E., Papanastasiou, G., Barzegar-Befroei, N., ... \& Lengyel, I. (2018). Peripheral retinal imaging biomarkers for Alzheimer's disease: a pilot study. Ophthalmic research, 59(4), 182-192.

8. Danesh-Meyer, H. V., Birch, H., Ku, J. F., Carroll, S., \& Gamble, G. (2006). Reduction of optic nerve fibers in patients with Alzheimer disease identified by laser imaging. Neurology, 67(10), 1852-1854.

9. Einarsdottir, A. B., Hardarson, S. H., Kristjansdottir, J. V., Bragason, D. T., Snaedal, J., \& Stefánsson, E. (2016). Retinal oximetry imaging in Alzheimer's disease. Journal of Alzheimer's Disease, 49(1), 79-83.

10. Frost, Shawn, Yogi Kanagasingam, Hamid Sohrabi, Janardhan Vignarajan, P. Bourgeat, Olivier Salvado, Victor Villemagne et al. "Retinal vascular biomarkers for early detection and monitoring of Alzheimer's disease." Translational psychiatry 3, no. 2 (2013): e233.

11. Gao, L., Liu, Y., Li, X., Bai, Q., \& Liu, P. (2015). Abnormal retinal nerve fiber layer thickness and macula lutea in patients with mild cognitive impairment and Alzheimer's disease. Archives of gerontology and geriatrics, 60(1), 162-167.

12. Liu, B., Rasool, S., Yang, Z., Glabe, C. G., Schreiber, S. S., Ge, J., \& Tan, Z. (2009). Amyloid-peptide vaccinations reduce $\beta$-amyloid plaques but exacerbate vascular deposition and inflammation in the retina of Alzheimer's transgenic mice. The American journal of pathology, 175(5), 2099-2110.

13. London, A., Benhar, I., \& Schwartz, M. (2013). The retina as a window to the brain-from eye research to CNS disorders. Nature Reviews Neurology, 9(1), 44.

14. Lu, Y., Li, Z., Zhang, X., Ming, B., Jia, J., Wang, R., \& Ma, D. (2010). Retinal nerve fiber layer structure abnormalities in early Alzheimer's disease: evidence in optical coherence tomography. Neuroscience letters, 480(1), 69-72.

15. Kesler, A., Vakhapova, V., Korczyn, A. D., Naftaliev, E., \& Neudorfer, M. (2011). Retinal thickness in patients with mild cognitive impairment and Alzheimer's disease. Clinical neurology and neurosurgery, 113(7), 523-526.

16. Santos, C. Y., Johnson, L. N., Sinoff, S. E., Festa, E. K., Heindel, W. C., \& Snyder, P. J. (2018). Change in retinal structural anatomy during the preclinical stage of Alzheimer's disease. Alzheimer's \& Dementia: Diagnosis, Assessment \& Disease Monitoring, 10, 196-209.

17. Polanco, J. C., Li, C., Bodea, L. G., Martinez-Marmol, R., Meunier, F A., \& Götz, J. (2018). Amyloid- $\beta$ and tau complexity-towards improved biomarkers and targeted therapies. Nature Reviews Neurology, 14(1), 22

18. Rohrschneider, K., Bültmann, S., \& Springer, C. (2008). Use of fundus perimetry (microperimetry) to quantify macular sensitivity. Progress in retinal and eye research, 27(5), 536-548.

\section{AUTHORS PROFILE}

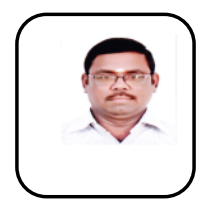

Mr. P.S.Ramesh pursuing his Doctorate in Computer Science and Engineering from Annamalai University. He has a vast experience in teaching and industry. His research interests include Image Processing, Transformation Techniques, Data warehouse and Data Mining, Big Data and IoT. He has published 9 research papers in referred journals. He is Life time member in CSI.

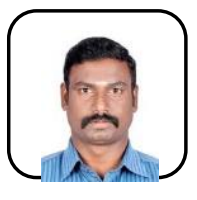

Dr. S. Arivalagan working as an Assistant Professor in the Department of Computer Science and Engineering. He is a member of Computer Society of India, Indian Society for Technical Education. His areas of interest include Image Processing, Soft Computing, Data Mining and Big Data Analytics.

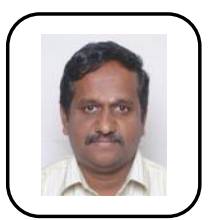

Dr. P. Sudhakar working as an Associate Professor in the Department of Computer Science and Engineering. He is a member of Computer Society of India, Institution of Electronics and Telecommunication Engineering, Institution of Engineers (India), Indian Science Congress Association, Indian Society for Technica Education, VLSI Society of India etc., His areas of interest include Software Engineering, Data Mining, and Big Data Analytics 